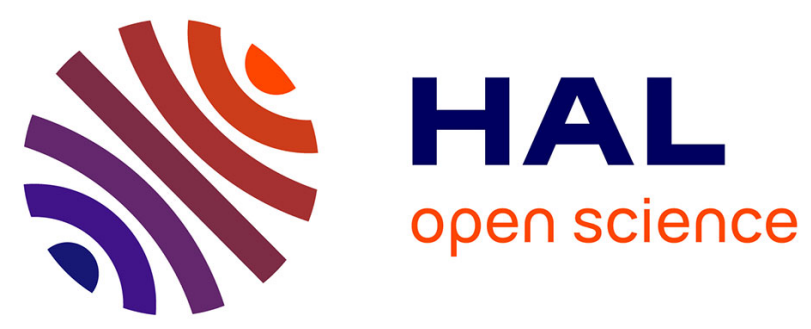

\title{
Pierres sculptées et meule gallo-romaines de Magnac-Laval (Haute-Vienne)
}

Franck Delage

\section{To cite this version:}

Franck Delage. Pierres sculptées et meule gallo-romaines de Magnac-Laval (Haute-Vienne). Gallia - Fouilles et monuments archéologiques en France métropolitaine, 1951, 9, pp.63-66. 10.3406/galia.1951.1298 . hal-01919970

\section{HAL Id: hal-01919970 \\ https://hal.science/hal-01919970}

Submitted on 3 Mar 2020

HAL is a multi-disciplinary open access archive for the deposit and dissemination of scientific research documents, whether they are published or not. The documents may come from teaching and research institutions in France or abroad, or from public or private research centers.
L'archive ouverte pluridisciplinaire HAL, est destinée au dépôt et à la diffusion de documents scientifiques de niveau recherche, publiés ou non, émanant des établissements d'enseignement et de recherche français ou étrangers, des laboratoires publics ou privés.

\section{(이) $\$$}

Distributed under a Creative Commons Attribution - NonCommercial - NoDerivatives $\mid 4.0$ 
Quant a ceux-ci - la tête colossale et le 'Terme -- on peut se demander s'ils ne seraient pas les "deux statues en grès. srossièrement travaillćes ", mises au jour prés de Montceau-les-Mines, ì Montsaint-Vincent, avant 1839, et que l'on croyail perdues. Ces sculptures nous semblaient, d'après le récit sommaire de la trouvaille fait par Monnier, devoir être plutòt attribućes au Moyen age ${ }^{8}$. Mais il faut peut-être réviser cette opinion. Sans doute l'occupation romaine n'est-elle pas encore prouvée en ce bourg par des dé('ouvertes incontestables ${ }^{9}$. Cependant, il paraît bien que Mont-Saint-Vineent soit autre chose qu'une création médićvale et que ce sommet, le plus haut du Chalonnais, couronné par une église dès le $\mathrm{x}^{\mathrm{e}}$ s. ainsi que par une forteresse 10 et par un chanip de fóre, ait été primitivement, comme celui de Gourdon, comme celui du Mont-Saint-Martin de Jaives el comme celui de Châtel-Moron: un petit oppidum celtique, ainsi que Bulliot l'avait plessenti, lorsqu'il écrivait son Essai sur le système défensif des Romains au pays ciluen ${ }^{11}$.

(8) Voir notre Chalonnais gallo-romain, 193\%, p. 202. P. Chandiaux, Eludes hist. sur Mont-St-Vincent, Lyon, 1900, p. 7, parle d'une " tète de stalue en grès du pays » qu'il attribue à un «dieu lare».

(9) D'après Jules Chevrier, la statuette en bronze du Musée de Châlon $\mathrm{n}^{\circ} 354$, aurait śté déterrée a Mont-Saint-Vincent. Mais nous n'avons pas confirmation du fait et la bonne foi de Chevrier a pu être surprise. Voir notre Catalogue du Musée, 1950, p. 44. L'œuvre (RErNach, Rép. st., IV, p. 404, no 1) semble pré-romaine ou bien très archaïsante. Plusieurs hameaux ont par contre donné des vestiges antiques, certains sur le territoire de la même commune.

(10) Cartulaire de Paray-le-Monial, publié par U. Chrvalier, Paris, $1890, n^{\circ}$ 6. Lours VII assiégea la forteresse en 1166 (Histoire du roi Loulis VII, édition Molinier, p. $173 \mathrm{sq.}$

(11) Bubliot, système défensif, p. 84. Cf. Iém. de la Soc. Eduenne, t. XXXIII, p. 355.
Quoi qu'il en soit, les sculptures dont nous venons de parler sont intéressantes en raison du caractère indigène très aceusé qu'elles présentent. Elles constituent en quelque sorte des témoins de la persistance du vicil esprit celtique après la conquête, et elles attestent l'existence d'ateliers locaux de tailleurs de pierre, ateliers analogues ì ceux des bronziers qui travaillaient dans la mème région de la Gaule.

J.ouis ARMAND-CALLIAT.

\author{
PIERRES SCULPTÉES \\ ET MEULE GALLO-ROMAINES \\ DE MAGiac-tavia. (IHALTE-VIENNE)
}

I. -- Une stèle funćraire se trouve depuis une soixantaine d'années dans le parc du château de Magnac-Iaval, où sa présence n'arait pas encore été signalée. C'est un bloc de granit local, haut de $1 \mathrm{~m}$. 50, large de $0 \mathrm{~m}$. 65 et épais de $0 \mathrm{~m}$. 40. La partic supérieure a la forme d'une pyramide à \& pans d'inégale larsour. Cette partic qui arait été séparée (soit par accident, soit volontairement) du corps de la stèle, a été rescellée avec du ciment. Il y a sà et là quelques parlies dégradées, mais l'ensemble reste assez net (fig. 1).

La face principale montre un personnage masculin debout, les pieds légèrement écartés. Il est vêtu d'une tunique longue qui descend jusqu'au mollet et forme trois plis sur le devant. Sur la tunique est un manteau de même longueur, agrafé sur l'épaule droite ct rejeté sur le côté gauche de façon à découvrir la partie antéricure du corps, sauf la poitrine qu'il couvre presque entièrement. Je bras gauche est replié sur la poitrine et la main, dont les cind doigts sont visibles, tient le bord replié du manteau qui 
vient de l'épaule droite et retombe le long du còté gauche. I.e bras droit est allongé et la main tient au bout des doigts un objet qui semble être une bourse (objet a peu près cylindrique, long de $0 \mathrm{~m} .17$ et large de $0 \mathrm{~m}, 10$ ). T.e pied gauche est casse; le droit est complet, mais la chaussure est indéterminable. Ia tête est écrasée; il n'en reste que le contour.

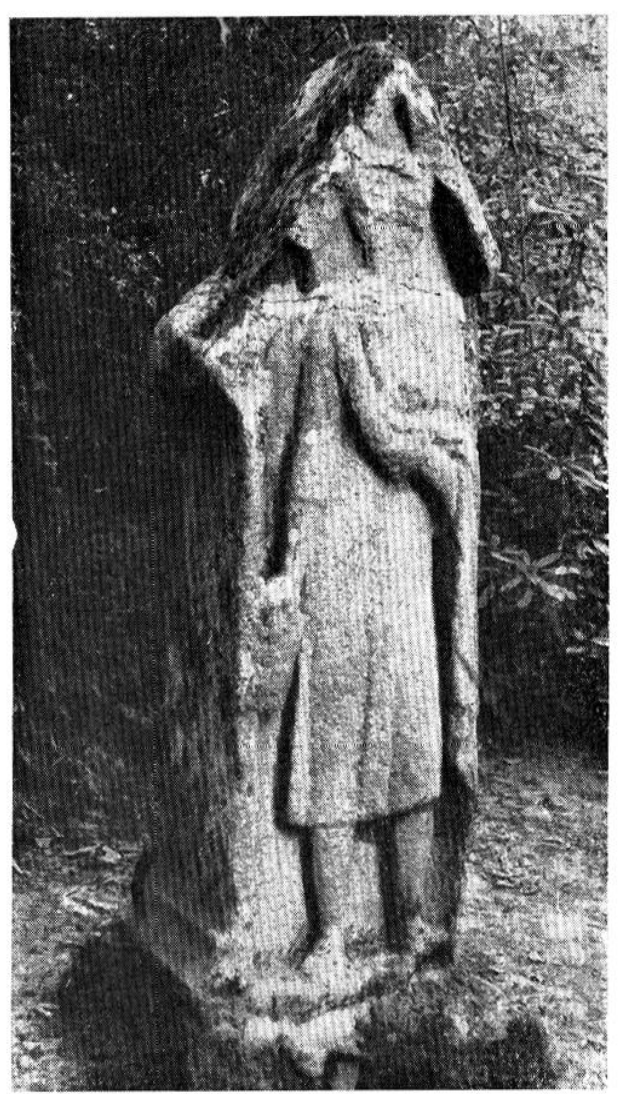

Fic. 1. - Slicle de Magnac-Iaval.

Fintre le bas de la jambe droite et le bord de la stile subsistent quelques lettres d'une inseriplion sur deux lignes. $A$ la premiere ligne, nous lisons nettement le mot SVI. Ides signes de la deuxiense ligne sont fails en majeure partie de lettres lices EEEEs. I) autre part, chitre le bas de la jambe gaucle et le bord verti- cal du manteau, nous avons discerné un signe ayant la forme d'un $Y$ avec queue terminée en crochet à gauche (hauleur : $0 \mathrm{~m}$. 035). Nous n'avons pu voir aucune autre lettre ou signe quelcongue. Cette inscription reste pour nous sans interprétation; disons seulement qu'elle paraît être funćraire

La face opposée de la stèle porte, dans le triangle supérieur, une têle humaine. masculine, croyons-nous ; cette tete blat isolée, sans corps et même sans buste. Elle a une chevelure abondante qui l'encadre à droite et à gauche el qui semble former deux pointes sur le haut. obliquemenl, des deux côtés de la tête; peutêtre est-ce deux cornes. In dessous, on voil une sorte de niche à sommet cintré (haut. : $0 \mathrm{~m} .62$. larg. : $0 \mathrm{~m}$. 29); prof. : () m. 04). Il est possible que celle niche nappartienne pas à l'état primitif du monument. Nous n'avons trouvé sur cette face aucune inscription. Ios faces latérales sont larges de $0 \mathrm{~m}$. 35 ; celle de ganche (eôté gauche du corps) a été diminuée verticalement, le long du bord du manteau, à partir du nivean de lépaule.

Sur la pente du fronton, côté gauche. une figure en forme diécusson la ligne supérieure étant divisie en deux ales de cercle concaves) resort en rolief de $0 \mathrm{~m} .015$. Est-ce une aldition moderne on bien un ornement antigue? Nous restons hésitants. Sur la penle du cotó droil, il a dû exister un omement somblable; au toucher on en sent puelques restiges.

Ia face latérale droile a une mouluration a la base du fronton et a la base de la stile. Tout au bas de la pente du fronton de celte face on roit un objet bombri ovale; ce pourrait être le vestige diune tète d'animal.

Jin résume; celte stele a. sur la face principale, une image funćraire aver inseription, qui devail concerner un marchanci ou un riche propriétaire foncier 
et, sur la face postérieure, une tête de divinité qui peut être Mercure. Elle était restée inconnue d'Fisperandieu, quand il prépara son Recucil des bas reliefs. Fin 1884, M. de Couronnel, qui possédait le château de Magnac-Laval, a signalé "une pierre liaute de $2 \mathrm{~m}$. environ, taillée sur les deux faces, représentant un personnage vêtu d'une tunique. On n'y voit pas d'inseription" 1. Nous sommes porlés a penser, malgré cette remarque, que cette indication concerne la stèle que nous déerivons ici. .t. de Couronnel a dû en faire l'acquisition ainsi que celle de divers objets antiques qu'il réunit diuns son pare où ils sont encore, conservés par l'actuel propriétaire, Y. Bancel 2. En 188', elle était "sur la terrasse du cercle militaire".

II. - Il à existé à Yagmale-Laval un autre monument antique qui a disparu danö la première muilié du $\mathrm{xt}^{\mathrm{e}}$ siècle. Il a été décrit en 1826 par un ćrudit limousin, C. Allou dans les termes suivants 3 : "pierre en forme d'autel portant sur une de ses faces une inseription latine dont on te peut lire gue les mots suivints: ...SESMVT ENIAE, et, au-dessous, D.S. C. ${ }^{4}$. Sur la face adjacente à celle qui porte l'inseription. (in distingue une figrue at temi-relief. dont on he voit que

(1) Notice sur Magnac-Laral, 1884, p. 8 .

(2) Parmi ces objels figure la borne milliaire plusieurs fois signalée (f. DEiatie, Bull. soc. arch. dhe Limousin, 193't t. LXXV, 1. 238-242, fig.), des coffres tuneraires et de: meules à bras, également gallo-romains, ainsi qu'un polissoir pour haches en pierre.

(3) Description des monuments de différents âges observés dans lo diportement de la Haule-Fienne, Limoges, 18:1, p. 311.

(4) Celle inscription a bie ensuite reproduite dans Trexisk, Mamuel d'eyigrophie, 1851. p. 103, no 36. - M. Aridst, Bull. Soc. arch. du Limousin, III. 184R. p. 30. - DLtochl: CRAI, 1860, p. 450, r: 3. - EspÉRaNDIEU, Inscriplions des Lémorices, 1890, p. 204. C.I.L., XIII, n' 1444. la parlic inféricure depuis la reinture jusquaux pieds, l'autel arant sté probablement mutile. Cette figure est revêtue d'une courte tunique qui ne descend que jusqu'aux genoux. La pierre est dans une silualion renversée et peut avoir 3 pieds do haul sur 2 pieds et demi de large sur sa plus grande facc." Cetle pierre était implantée dans la rue conduisant "de l'église aux ruines de l'ancion château". De Couronnel précise: " elle selvit très longtemps de borne daus un carrefour ... et ne fut dóterrie qu'en 1820, sur la demande de celui qui lavait décomverte". Il assure qu'un antiquare avail pu "y recomaititre les pieds ailés d'un llercure s.

Ge monumenl est assurément distinct de celui que nous arons décrit plus haut : ni les dimensions ni ia figure ne sont identiques, tout en présentant les ressemblances générates des stìles de même épocue.

III. - Parmi les objets antiques que II. de Couromel arait recueillis dans les environs de Magnac-laval et qui se trouvent heureusement encole dins le pare

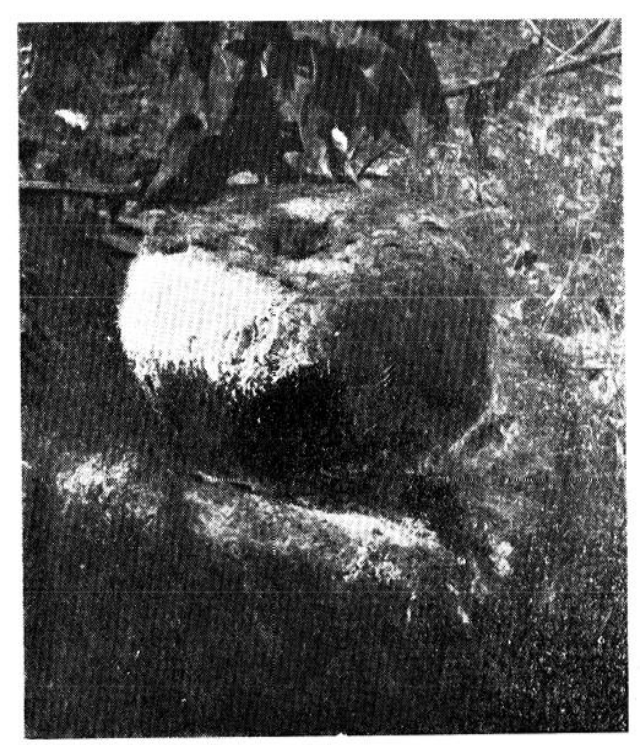

FIG. 2. - Meule complète. 

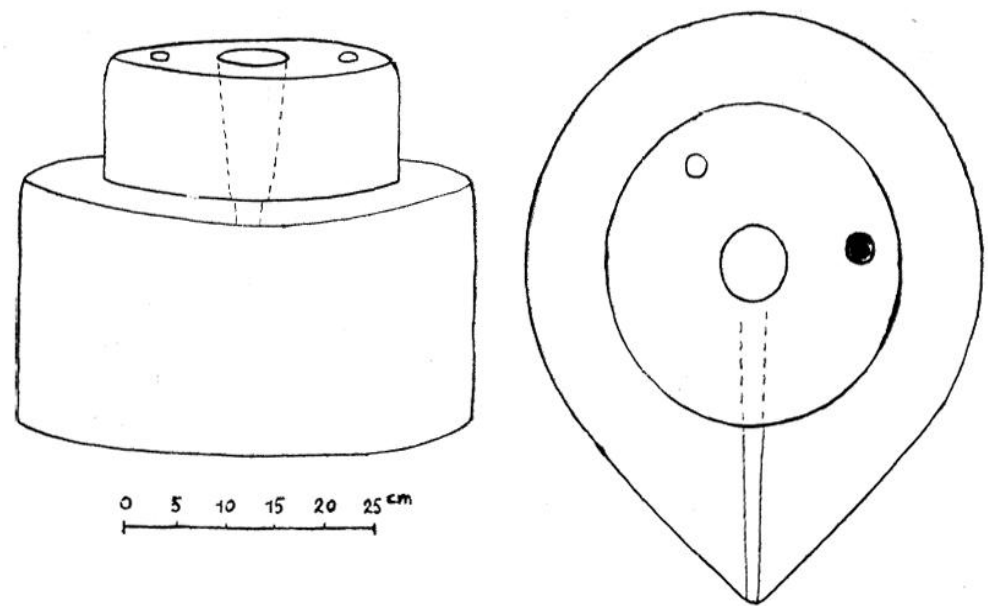

Fir. 3 et 4. - Meule : les deux parties diam. inf. $0^{m ! 6}$ ), $A$ droite : le catillus.

du chàteau, nous avons remarqué une meule à bras qui mérite d'être signalée. wlle a l'intérêt d'être complète, ce qui est extremement rare (fig. 2 ).

I,a partic supérieure, c'est-ì-dire la partic mobile (calillus) est haute de 0) $\mathrm{m}$. 11 et large de $0 \mathrm{~m}$. 30 (fig. 3). Le lrou vertical perforé au centre pour la descente du grain est large de $0 \mathrm{~m} .07$ à l'orifice supérieur; il va en se rétrécissant progressivement jusqu'a la face inférieure. Sur le dessus, on voit deux petits trous placés près du bord: la étaient fixés les blas servant a donner le mouvement au broyeur; un de ces trous contient les resles d'une tige de fer.

Ja meule dormante (mota), sur laquelle se pose la meule mobile, est large de () $\mathrm{m}$. 46 et haute de $0 \mathrm{~m}$. 25. Tandis que la dormante est cylindrique, la base présente une arancée accentuéc dans laquelle cst creusé le canal d'éracuation de la farine depuis le centre jusqu'au bord. I.es deux éléments étant scellés l'un sur l'autre, la largeur du canal n'est visible qu'au voisinage du bord, ou elle a seulement $0 \mathrm{~m} .025$; il cst probable qu'au centre la dimension r'épond à celle de l'orifice inféricur de l'arrivage des grains à broyer (fig. 1).
On ne peut guère attribuer qu’à l'époque gallo-romaine ce moulin, qui était à usage domestique, étant donné ses modestes dimensions, répondant bien à celles des meules découvertes dans les habitats de la Gaule romaine.

† Franck I)ELAGE.

JERMES DOUBLE TROUVÉ JEN NARBONNAISE

Au Sud-Est du département de l'Aude, rans les Corbières, une sculplure vient d'ètre découverte. Cette reuvre, en mauvais etat et assez fruste, n'aurail motivé qu'une mention afin de ne pas augmenter le nombre trop important des sculptures classées comme d'origine inconnue; mais, a son sujet, quelques points méritent cxamen.

Le licu de la découverte lía pas été précisé avec rigueur mais trois localités sont à retenir: Coustouge, Saint-Victor et surtout Fontjoncouse ćanton de Durban). Le nom de Coustouge ne révèle qu'un hypothétique Costuvium ${ }^{1}$, SaintVictor aflirme un vocable du Moyen âge,

(1) Sanartiks, Géographic historique du département de l'Aude. 